\title{
Improving the Performance of Fuzzy Rule-Based Classification Systems with Interval-Valued Fuzzy Sets and Genetic Amplitude Tuning
}

\author{
José Antonio Sanz ${ }^{\mathrm{a}, *}$, Alberto Fernández ${ }^{\mathrm{b}}$, Humberto Bustince ${ }^{\mathrm{a}}$, Francisco \\ Herrera $^{\mathrm{b}}$ \\ ${ }^{a}$ Dept. of Automatic and Computation, Public University of Navarra, Spain \\ ${ }^{b}$ Dept. of Computer Science and Artificial Intelligence, CITIC-UGR (Research Center \\ on Information and Communications Technology), University of Granada, Spain
}

\begin{abstract}
Among the computational intelligence techniques employed to solve classification problems, Fuzzy Rule Based Classification Systems (FRBCSs) are a popular tool because of their interpretable models based on linguistic variables, which are easier to understand for the experts or end-users.

The aim of this paper is to enhance the performance of FRBCSs by extending the Knowledge Base with the application of the concept of IntervalValued Fuzzy Sets (IVFSs). We consider a post-processing genetic tuning step that adjusts the amplitude of the upper bound of the IVFS to contextualize the fuzzy partitions and to obtain a most accurate solution to the problem.

We analyze the goodness of this approach using two basic and well-known fuzzy rule learning algorithms, the Chi et al.'s method and the Fuzzy Hybrid Genetics-Based Machine Learning algorithm. We show the improvement achieved by this model through an extensive empirical study with a large collection of data-sets.
\end{abstract}

Keywords: Fuzzy Rule-Based Classification Systems, Interval-Valued Fuzzy Sets, Tuning, Genetic Algorithms

\footnotetext{
*Corresponding author. Tel:+34-948169839. Fax:+34-948168924

Email addresses: joseantonio.sanz@unavarra.es (José Antonio Sanz ), alberto@decsai.ugr.es (Alberto Fernández), bustince@unavarra.es (Humberto Bustince), herrera@decsai.ugr.es (Francisco Herrera)
} 


\section{Introduction}

Fuzzy Rule-Based Classification Systems (FRBCS)[28] is an useful tool to deal with the classification problem. They are widely employed because of their capability to built a linguistic model interpretable to the users and the possibility of mixing different information as the one coming from expert knowledge and information coming from mathematical models or empiric measures. The significance of FRBCSs can be observed by the high quantity of real applications in which they have been applied including, but not limited to, detection of intrusions [41], medical applications [1, 43], Shewhart control charts [46] or newspaper demand prediction [11], among others.

As we state above, one of the main advantages of the FRBCSs is the high interpretability of the output model. However, the disadvantage of these systems is their lack of accuracy when dealing with some complex systems, due to the inflexibility of the concept of linguistic variable, which imposes hard restrictions to the fuzzy rule structure [6]. For example, sometimes when the classes are overlapped, we have not exact knowledge about the membership degree of some elements to the fuzzy sets that characterize the attributes defining the class. This fact suggests to represent the membership degrees of the objects to the fuzzy set by means of an interval. That is, to employ the Interval-valued Fuzzy Sets (IVFSs) to characterize the linguistic labels that compound the attributes of the problem.

In $[31,34,35,36,37]$ the authors show that IVFSs are a particular case of Type-2 Fuzzy Sets. The latter are an old construct suggested by Zadeh in [45]. They are natural concepts addressing the apparent paradox, faced by standard fuzzy sets, of modeling imprecise concepts using precise membership functions (MFs) (see foreword by D. Dubois in [9]). IVFSs allow to take into account the effect of the ignorance of the expert in the MF definition (see [10]). Their construction may be done in different ways, like the ones given by Bustince [7, 8], Liang and Mendel [32] and Tizhoosh [40], among others.

The aim of this paper is to enhance the performance of FRBCSs by extending the Knowledge Base (KB) with the application of the concept of IVFSs. The main novelty of our approach is the use of IVFSs in the Fuzzy Reasoning Method (FRM), which will allow us to handle the uncertainty that is inherent to the definition process of the MFs. In this sense, we want to stress that the learning algorithms we will use are those proposed in the original works.

Furthermore, we consider the application of a post-processing genetic 
tuning step in order to adjust the amplitude of the upper bound of the IVFS to contextualize the fuzzy partitions and to obtain a most accurate solution to the problem. This issue is due to the fact that the length of the intervals representing the membership of each element to the IVFS does not need to be the same, because the expert does not have the same ignorance in the definition of the MFs to each element.

In the specialized literature we can find different proposals that performs a genetic tuning to the $\mathrm{KB}$ of the fuzzy system, both applied to modeling and classification problems $[2,12,16]$. These approaches try to induce a better cooperation among the rules by acting on one or two different model components: the fuzzy partition parameters stored in the Data Base (DB) and the Rule Base (RB). The amplitude of the support of each label determines the specificity and involves a potential accuracy improvement, since it could determine the best covering region of the labels. For example, in [3] the authors propose to tune the MF of each label adjusting both the position (performing lateral displacements) and the length of the support (performing variations in the amplitude).

We analyze the goodness of this approach using two basic and wellknown fuzzy rule learning algorithms, the Chi et al.'s method [13] and the Fuzzy Hybrid Genetics-Based Machine Learning (FH-GBML) algorithm by Ishibuchi and Yamamoto [30]. We have chosen [13] since it is one of the most widely used algorithms, and [30] due to the fact that it is a more robust fuzzy rule extraction algorithm which allows us to obtain a higher quality RB [33]. Both algorithms are available within the KEEL software tool [4] (http://www.keel.es).

To carry out the empirical analysis, we have selected 24 data-sets from UCI repository [5], using the standard accuracy rate as evaluation measure. All the results from the comparisons appear along the text in different tables showing the improvements obtained by adapting to the IVFSs the two cited methods. To strengthen further our experimental study, we also compare our results with those obtained by a recent method proposed by J. Dombi and Z. Gera in [19] (called along the paper GAGRAD method). Furthermore, our experimental results are supported by means of a strong statistical study using non-parametric tests as stated in [17, 23, 22].

In order to do that, this paper is organized as follows. First, we present in Section 2 the basic concepts employed in the paper for FRBCSs and IVFSs. In Section 3 we describe in detail the IVFSs model, showing the modifications introduced in the FRM, and the proposal to tune the amplitude of the upper 
bound of the IVFSs. Section 4 shows our experimental framework and the experimental analysis carried out. Finally, some concluding remarks are presented in Section 5.

\section{Preliminaries: Fuzzy Rule-Based Classification Systems and Interval- valued Fuzzy Sets}

In this section we carry out a brief introduction of FRBCSs and the IVFSs theory. First, we will describe the type of fuzzy rules used in this work, together with the rule weight and the FRM. Next, we will present the rule learning algorithms used: the Chi et al.'s rule generation approach [13] and the FH-GBML algorithm [30]. Finally, we will define some basic concepts of the IVFSs theory.

\subsection{Fuzzy Rule-Based Classification Systems}

To deal with the classification problem there exists a lot of techniques in the Data Mining field. Among them, FRBCSs provides an interpretable model by means of the use of linguistic labels in their rules.

Consider $\mathrm{m}$ labeled patterns $x_{p}=\left(x_{p 1}, \ldots, x_{p n}\right), p=1,2, \ldots, m$ where $x_{p i}$ is the $i$ th attribute value $(i=1,2, \ldots, n)$. We have a set of linguistic values (and their MFs) describing each attribute. We use fuzzy rules of the following form:

Rule $R_{j}$ : If $x_{1}$ is $A_{j 1}$ and $\ldots$ and $x_{n}$ is $A_{j n}$ then Class $=C_{j}$ with $R W_{j}$

where $R_{j}$ is the label of the $j$ th rule, $x=\left(x_{1}, \ldots, x_{n}\right)$ is an n-dimensional pattern vector, $A_{j i}$ is an antecedent fuzzy set representing a linguistic term, $C_{j}$ is a class label, and $R W_{j}$ is the rule weight [27]. Specifically, the rule weight is computed using the Penalized Certainty Factor (PCF) defined in [29] as:

$$
P C F_{j}=\frac{\sum_{x_{p} \in \text { Class } C_{j}} \mu_{A_{j}}\left(x_{p}\right)-\sum_{x_{p} \notin \text { Class } C_{j}} \mu_{A_{j}}\left(x_{p}\right)}{\sum_{p=1}^{m} \mu_{A_{j}}\left(x_{p}\right)}
$$


If $x_{p}=\left(x_{p 1}, \ldots, x_{p n}\right)$ is a new pattern and if $\mathrm{L}$ denotes the number of rules in the $\mathrm{RB}$ and $\mathrm{M}$ the number of classes of the problem, the steps of the FRM [14] are the following:

1. Matching degree, that is, the strength of activation of the if-part for all rules in the $R B$ with the pattern $x_{p}$. To compute it we employ a product or minimum T-norm.

$$
\mu_{A_{j}}\left(x_{p}\right)=T\left(\mu_{A_{j 1}}\left(x_{p 1}\right), \ldots, \mu_{A_{j n}}\left(x_{p n}\right)\right), \quad j=1, \ldots, L .
$$

2. Association degree. To compute the association degree of the pattern $x_{p}$ with the $M$ classes according to each rule in the $R B$. When using rules like (1) this association degree only refers to the consequent class of the rule (i.e. $\left.k=\operatorname{Class}\left(R_{j}\right)\right)$.

$$
\mathrm{b}_{j}^{k}=h\left(\mu_{A_{j}}\left(x_{p}\right), R W_{j}^{k}\right) \quad k=1, \ldots, M, \quad j=1, \ldots, L .
$$

3. Pattern classification soundness degree for all classes. We use an aggregation function that combines the positive degrees of association calculated in the previous step.

$$
Y_{k}=f\left(b_{j}^{k}, j=1, \ldots, L \text { and } b_{j}^{k}>0\right), \quad k=1, \ldots, M .
$$

4. Classification. We apply a decision function $F$ over the soundness degree of the system for the pattern classification for all classes. This function will determine the class label $l$ corresponding to the maximum value.

$$
F\left(Y_{1}, \ldots, Y_{M}\right)=l \quad \text { such that } \quad Y_{l}=\left\{\max \left(Y_{k}\right), k=1, \ldots, M\right\} .
$$

\subsection{Chi et al. rule generation algorithm}

To generate the fuzzy RB this FRBCSs design method determines the relationship between the variables of the problem and establishes an association between the space of the features and the space of the classes by means of the following steps:

1. Establishment of the linguistic partitions. Once the domain of variation of each feature $A_{i}$ is determined, the fuzzy partitions are computed.

2. Generation of a fuzzy rule for each example $x_{p}=\left(x_{p 1}, \ldots, x_{p n}, C_{p}\right)$. For this it is necessary: 
2.1 To compute the matching degree $\mu\left(x_{p}\right)$ of the example to the different fuzzy regions using a conjunction operator (usually modeled with a minimum or product T-norm).

2.2 To assign the example $x_{p}$ to the fuzzy region with the greatest membership degree.

2.3 To generate a rule for the example, whose antecedent is determined by the selected fuzzy region and whose consequent is the label of class of the example.

2.4 To compute the rule weight.

We must remark that rules with the same antecedent can be generated during the learning process. If they have the same class in the consequent we just remove one of the duplicated rules, but if they have a different class only the rule with the highest weight is kept in the RB.

\subsection{Fuzzy hybrid genetic based machine learning rule generation algorithm}

Different Genetic Fuzzy Systems have been proposed in the specialized literature for designing fuzzy rule-based systems in order to avoid the necessity for linguistic knowledge from domain experts [15, 21, 25, 38].

The basis of the method described here, the FH-GBML algorithm [30], consists in a Pittsburgh approach where each rule set is handled as an individual. It also contains a Genetic Cooperative Competitive Learning (GCCL) approach (an individual represents an unique rule), which is used as a kind of heuristic mutation for partially modifying each rule set, because of its high search ability to efficiently find good fuzzy rules.

This method simultaneously uses four fuzzy set partitions for each attribute, as shown in Figure 1. As a result, each antecedent attribute is initially associated with 14 fuzzy sets generated by these four partitions as well as a special "do not care" set, i.e., 15 in total.

The main steps of this algorithm are described below:

Step 1: Generate $N_{\text {pop }}$ rule sets with $N_{\text {rule }}$ fuzzy rules.

Step 2: Calculate the fitness value of each rule set in the current population.

Step 3: Generate $\left(N_{\text {pop }}-1\right)$ rule sets by selection, crossover and mutation in the same manner as the Pittsburgh-style algorithm. Apply a single iteration of the GCCL-style algorithm (i.e., the rule generation and the replacement) to each of the generated rule sets with a pre-specified probability. 

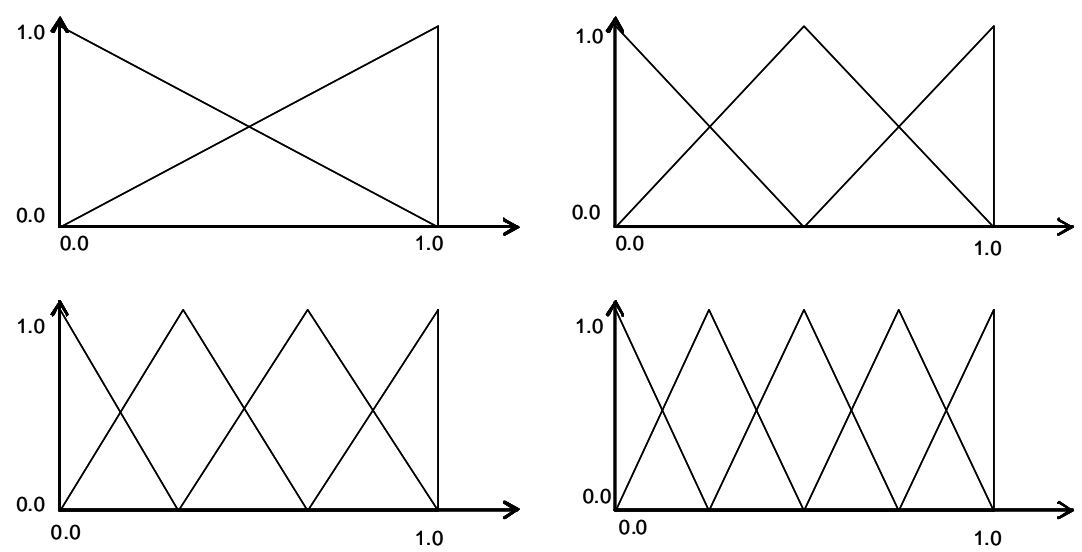

Figure 1: Four fuzzy partitions for each attribute membership function

Step 4: Add the best rule set in the current population to the newly generated $\left(N_{p o p}-1\right)$ rule sets to form the next population.

Step 5: Return to Step 2 if the pre-specified stopping condition is not satisfied.

Next, we will describe every step of the algorithm:

- Initialization: $N_{\text {rule }}$ training patterns are randomly selected. Then, a fuzzy rule from each of the selected training patterns is generated by choosing probabilistically (as shown in (7)) an antecedent fuzzy set from the 14 candidates $B_{k}(k=1,2, \ldots, 14)$ (see Figure 1) for each attribute. Then each antecedent fuzzy set of the generated fuzzy rule is replaced with don't care using a pre-specified probability $P_{d o n^{\prime} t}$ care .

$$
P\left(B_{k}\right)=\frac{\mu_{B_{k}}\left(x_{p i}\right)}{\sum_{j=1}^{14} \mu_{B_{j}}\left(x_{p i}\right)}
$$

- Fitness computation: The fitness value of each rule set $S_{i}$ in the current population is calculated as the number of correctly classified training patterns by $S_{i}$. For the GCCL approach the computation follows the same scheme, counting the number of correct hits for each single rule.

- Selection: It is based on binary tournament. 
- Crossover: The substring-wise and bit-wise uniform crossover are applied in the Pittsburgh part. In the case of the GCCL part only the bit-wise uniform crossover is considered.

- Mutation: Each fuzzy partition of the individuals is randomly replaced with a different fuzzy partition using a pre-specified mutation probability for both approaches.

For more details about this proposal, please refer to [30].

\subsection{Interval-valued Fuzzy Sets}

As we stated in the introduction, in many cases the MF of a fuzzy set representing a linguistic term can not be defined unequivocally on the basis of available information. The selection of the MF is very difficult to carry out without losing any information. IVFSs allow to take into account this shortage of information because they assign as membership an interval instead of a single number. These sets were born in 1970's with the work of Sambuc (see [39]). In the 80's, Gorzalczany and Turksen denoted these sets for the first time as IVFSs (see [24, 42]).

We must point out that, in the literature, IVFSs are also called Interval Type-2 Fuzzy Sets. Liang and Mendel have carried out a deep study about these sets in [32] and $\mathrm{Wu}$ and Mendel gave uncertainty measures for these sets in [44]. Furthermore, a comparative study about the different extensions of fuzzy sets can be found in [18].

Definition 1. An Interval-valued Fuzzy Set (IVFS) A on the universe $U \neq \emptyset$ is given by:

$$
A=\{(u, A(u)) \mid u \in U\}
$$

where

$$
A(u)=[\underline{A}(u), \bar{A}(u)] \in L([0,1])
$$

being

$$
L([0,1])=\left\{\mathbf{x}=[\underline{x}, \bar{x}] \mid(\underline{x}, \bar{x}) \in[0,1]^{2} \text { and } \underline{x} \leq \bar{x}\right\}
$$

Obviously, $A(u)=[\underline{A}(u), \bar{A}(u)]$ is the membership degree of $u \in U$. Figure 2 depicts two examples of IVFSs: the interval $\left[\underline{A_{j}}(u), \overline{A_{j}} u\right]$ and not a number from $[0,1]$ is assigned as the membership to each element $u \in U$.

Referring to the earlier considerations, it seems that the idea of an IVFS, from the point of view of practice, is a sufficiently complete generalization 


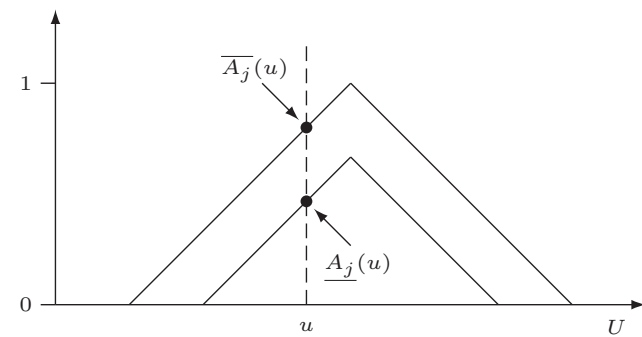

(a) Generic IVFS

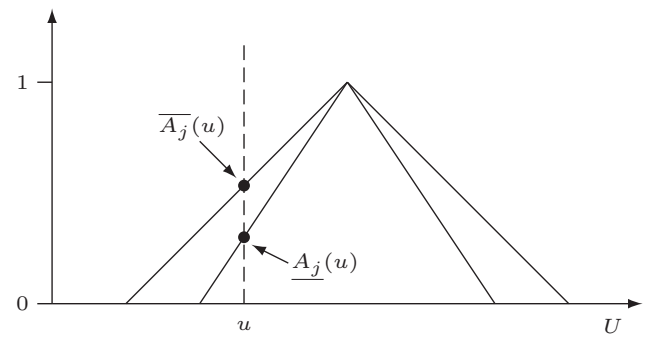

(b) IVFS employed in this work

Figure 2: Examples of IVFSs

of the concept of fuzzy set. On the other hand, it is not such a conspicuous generalization that could have a considerably negative influence on the effectiveness of approximate inference, based on IVFSs, which will be presented in subsection 3.2.

\section{Fuzzy Rule-Based Classification Systems based on Interval-Valued Fuzzy Sets and Genetic Amplitude tuning}

The previous considerations suggest the use of IVFSs to improve the performance of FRBCSs. In order to do that, we model the fuzzy partitions by means of IVFSs, the rule weight will be composed by two numbers (Subsection 3.1) and we perform changes in the FRM (Subsection 3.2). In the tuning step the amplitude of the upper bound of each IVFS is modified to contextualize the fuzzy partitions to the problem to solve (Subsection 3.3).

\subsection{Interval-Valued Fuzzy Sets based fuzzy partition}

From any KB, which can be generated by an ad-hoc method, we include the IVFSs model by adding an upper bound for each fuzzy partition, centered in the maximum of the MF and with a higher amplitude.

In Figure 3 we can see an example of a linguistic variable represented by 3 labels (IVFSs) in the initial state. The solid lines represent the lower bounds $\left(A_{j}\right)$ and the dashed lines represent the upper bounds $\left(\overline{A_{j}}\right)$. We must point out that in the initial situation the amplitude of the upper bound is $50 \%$ greater than the one of the lower bound (see Subsection 3.3.1). We must remark that we note "upper" and "lower" bounds referring to the corresponding fuzzy labels, following the standard notation of the IVFSs literature. 


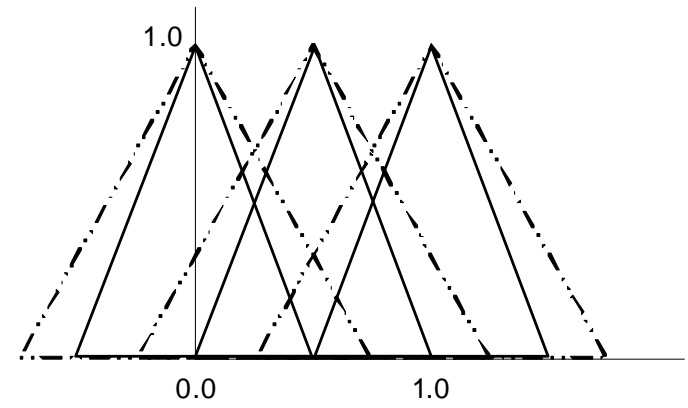

Figure 3: Example of the interval-valued fuzzy sets. The solid line is the lower bounds $\left(A_{j}\right)$ and the dashed line is the upper bounds $\left(\overline{A_{j}}\right)$

We will employ rules in the form presented in Subsection 2.1 where each $A_{j i}$ will be an IVFS instead of a fuzzy set.

\subsection{Fuzzy reasoning method}

The modifications in the structure of the fuzzy labels, also implies an extension of the original FRM used for classifying new patterns. The general model of fuzzy reasoning for classification, presented in the subsection 2.1 , will be modified in the following way (being $x_{p}=\left(x_{p 1}, \ldots, x_{p n}\right)$ a new pattern):

1. We have two matching degrees because we are working with an interval, each one will be associated with the lower and the upper bound respectively and will be calculated applying a T-norm in the following way:

$$
\begin{array}{ll}
\mu_{L} A_{j}\left(x_{p}\right)=T\left(\underline{A_{j 1}}\left(x_{p 1}\right), \ldots, \underline{A_{j n}}\left(x_{p n}\right)\right), & j=1, \ldots, L . \\
\mu_{U} A_{j}\left(x_{p}\right)=T\left(\overline{A_{j 1}}\left(x_{p 1}\right), \ldots, \overline{A_{j n}}\left(x_{p n}\right)\right), & j=1, \ldots, L .
\end{array}
$$

We apply a T-norm to both lower and upper bounds. Therefore, the matching degrees obtained form the following interval: $\left[\mu_{L} A_{j}\left(x_{p}\right), \mu_{U} A_{j}\left(x_{p}\right)\right]$.

2. As association degree we take the mean of the product of the matching degree by the rule weight, which is composed by two numbers, associated to the lower and the upper bound respectively. The rule weights will be denoted as $P C F_{L j}$ and $P C F_{U j}$ and their computation will be 
done following the Expression (2), considering the lower and the upper bounds as the terms in each case. That is:

$$
\begin{aligned}
& P C F_{L j}=\frac{\sum_{x_{p} \in C l a s s C_{j}} \underline{A_{j}}\left(x_{p}\right)-\sum_{x_{p} \notin C l a s s C_{j}} \underline{A_{j}}\left(x_{p}\right)}{\sum_{p=1}^{m} \underline{A_{j}}\left(x_{p}\right)}
\end{aligned}
$$

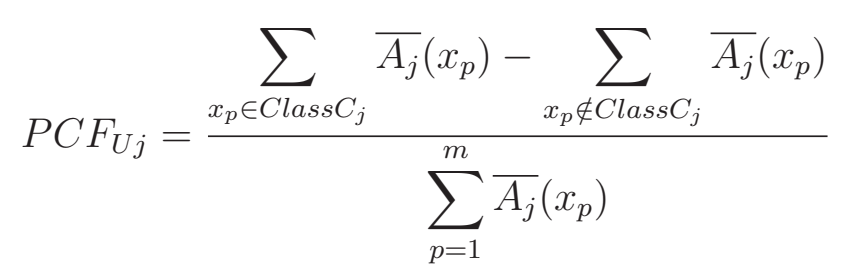

The final association degree will be computed as follows:

$$
\begin{array}{r}
b_{j}^{k}=\frac{\mu_{L} A_{j}\left(x_{p}\right) * P C F_{L} j^{k}+\mu_{U} A_{j}\left(x_{p}\right) * P C F_{U} j^{k}}{2} \\
k=1, \ldots, M, \quad j=1, \ldots, L .
\end{array}
$$

At this point we have already a single value associated to the class. According to this, we can apply the rest of the algorithm in the same way than in the general FRM presented in [14].

\subsection{Genetic Tuning of the Fuzzy Rule-Based Classification Systems}

The goal of basic linguistic fuzzy modeling methods is to determine the set of fuzzy rules that compounds the RB of the model. The MFs, usually obtained by experts knowledge or by a normalization process, remains fixed in the rule set derivation process. So, the fuzzy partitions are not adapted to the context of each variable. Furthermore, the rule set derivation process can include some rules with bad performance and hence the cooperative behavior of the rules may not be optimal.

In order to improve the accuracy of the FRBCSs we propose to adjust the MFs making variations in the support of the upper bounds of the IVFSs by means of a Genetic Algorithm (GA); specifically we consider the use of $\mathrm{CHC}$ algorithm [20]. We have named this methodology as Genetic Amplitude Tuning, and it is described in the remaining of this section. 


\subsubsection{Genetic Amplitude Tuning}

We have added the upper bound of the IVFSs with a fixed amplitude but, as the data distribution does not need to be uniform, the support can be different for each linguistic label. We apply a post-processing genetic tuning step in order to improve the behavior of the FRBCSs performing slight changes of the amplitude in the upper bound of the IVFSs. We must point out that the amplitude of lower bound remains fixed.

The modification of the amplitude is given by a number within the interval $[0,1]$, that is, from the overlapping of both bounds (value 0 ) to twice the amplitude of the upper with respect to the lower bound (value 1). The amplitude of the upper bound will be uniformly increased according to intermediate values being 0.5 the initial situation, that is, when the amplitude of the upper bound is $50 \%$ greatter than of the lower bound. This situations are depicted in Figure 4.

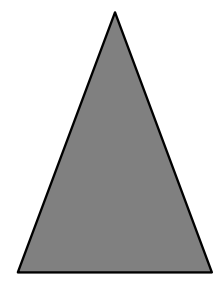

a) Gene $=0.0$

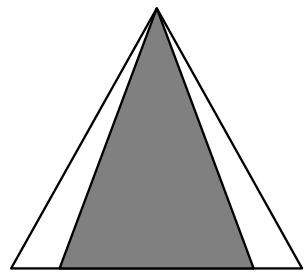

b) $\mathrm{Gene}=0.5$

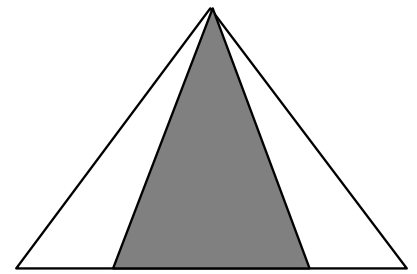

c) Gene $=1.0$

Figure 4: Gene values representation in the genetic amplitude tuning. a) Upper and lower bounds are overlapped. b) Initial situation. c) Upper bound amplitude is twice than the one of the lower bound

\subsubsection{Genetic algorithm for tuning: the $\mathrm{CHC}$ algorithm}

GAs has been widely used to derive fuzzy systems. In this paper, we will consider the use of the $\mathrm{CHC}$ algorithm [20] because it presents a good trade-off between diversity and convergence, being a good choice in complex problems. The genetic model makes use of a mechanism of "Selection of Populations". $M$ parents and their corresponding offspring are put together to select the best $M$ individuals to take part of the next population (with $M$ being the population size).

To provoke diversity in the population, the $\mathrm{CHC}$ approach makes use of an incest prevention mechanism and a restarting approach, instead of the well 
known mutation operator. The components needed to design this process are explained below:

1. Coding Scheme: A real coding is considered, where each gene of the chromosome represents the amplitude modifier as defined above. Thus, there are as many genes as fuzzy partitions in the DB.

2. Chromosome Evaluation: We use as fitness function the standard accuracy rate.

3. Initial Gene Pool: The initial pool is obtained with the first individual having all genes with value '0.5' (the initial FRBCS). The second and the third individuals having all genes with values 0 and 1 respectively, whereas the remaining individuals are generated at random in $[0,1]$.

4. Crossover Operator: We consider the Parent Centric BLX (PCBLX) operator [26], which is based on the BLX- $\alpha$. Figure 5 depicts the behavior of these kinds of operators.

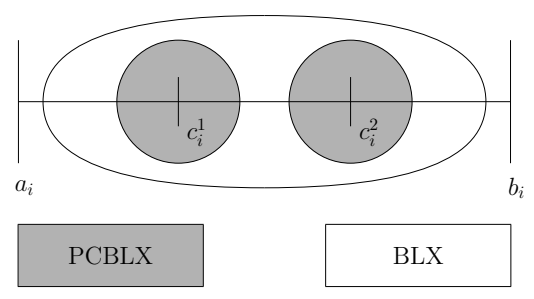

Figure 5: Scheme of the behavior of the BLX and PCBLX operators

PCBLX is described as follows. Let us assume that $X=\left(x_{1} \cdots x_{n}\right)$ and $Y=\left(y_{1} \cdots y_{n}\right),\left(x_{i}, y_{i} \in\left[a_{i}, b_{i}\right] \subset \Re, i=1 \cdots n\right)$, are two realcoded chromosomes that are going to be crossed. PCBLX operator generates the two following offspring:

- $O_{1}=\left(o_{11} \cdots o_{1 n}\right)$, where $o_{1 i}$ is a randomly (uniformly) chosen number from the interval $\left[l_{i}^{1}, u_{i}^{1}\right]$, with $l_{i}^{1}=\max \left\{a_{i}, x_{i}-I_{i}\right\}, u_{i}^{1}=$ $\min \left\{b_{i}, x_{i}+I_{i}\right\}$, and $I_{i}=\left|x_{i}-y_{i}\right|$.

- $O_{2}=\left(o_{21} \cdots o_{2 n}\right)$, where $o_{2 i}$ is a randomly (uniformly) chosen number from the interval $\left[l_{i}^{2}, u_{i}^{2}\right]$, with $l_{i}^{2}=\max \left\{a_{i}, y_{i}-I_{i}\right\}$ and 


$$
u_{i}^{2}=\min \left\{b_{i}, y_{i}+I_{i}\right\}
$$

On the other hand, the incest prevention mechanism will be only considered in order to apply the PCBLX operator. In our case, two parents are crossed if their hamming distance divided by 2 is above a predetermined threshold, $L$. Since we consider a real coding scheme, we have to transform each gene considering a Gray Code (binary code) with a fixed number of bits per gene (BITSGENE), that is determined by the system expert. In this way, the threshold value is initialized as:

$$
L=(\# \text { Genes } \cdot \text { BITSGENE) } / 4.0
$$

where \#Genes stands for the total length of the chromosome. Following the original CHC scheme, $L$ is decremented by one (BITSGENE in this case)when there are no new individuals in the next generation.

5. Restarting approach: When the threshold value is lower than zero, all the chromosomes are regenerated at random within the interval $[0,1]$. Furthermore, the best global solution found is included in the population to increase the convergence of the algorithm.

\section{Experimental Study}

In this section, we will first present the experimental framework and the configuration parameters of the learning algorithms selected for this study and then we will present the empirical study carried out.

\subsection{Experimental Set-Up}

We have selected twenty-four data-sets from UCI repository [5]. The data are summarized in Table 1, showing the data-set name, the number of examples (\#Ex.), attributes (\#Atts.) and classes (\#Class.).

To carry out the different experiments we consider a 5-folder cross-validation model, i.e., 5 random partitions of data with a $20 \%$, and the combination of 4 of them (80\%) as training and the remaining one as test. For each data-set we consider the average results of the five partitions.

We will apply the same configuration for both FRBCS approaches (Chi and FH-GBML), consisting in product T-norm as conjunction operator, together with Penalized Certainty Factor approach [29] for the rule weight and 
Table 1: Summary description for the data-sets of the study

\begin{tabular}{|l|c|c|c|}
\hline Data-set & \#Ex. & \#Atts. & \#Class. \\
\hline Australian & 690 & 14 & 2 \\
\hline Autos & 159 & 25 & 5 \\
\hline Balance & 625 & 4 & 3 \\
\hline Bupa & 345 & 6 & 2 \\
\hline Car & 1728 & 6 & 4 \\
\hline Cleveland & 297 & 13 & 5 \\
\hline Contraceptive & 1473 & 9 & 3 \\
\hline Crx & 125 & 15 & 2 \\
\hline Ecoli & 336 & 7 & 8 \\
\hline German & 1000 & 20 & 2 \\
\hline Glass & 214 & 9 & 6 \\
\hline Haberman & 306 & 3 & 2 \\
\hline Hepatitis & 155 & 19 & 2 \\
\hline Iris & 150 & 4 & 3 \\
\hline Magic & 1902 & 10 & 2 \\
\hline New-Thyroid & 215 & 5 & 3 \\
\hline Page-blocks & 548 & 10 & 5 \\
\hline Penbased & 1099 & 16 & 10 \\
\hline Pima & 768 & 8 & 2 \\
\hline Ring & 768 & 8 & 2 \\
\hline Tae & 151 & 5 & 3 \\
\hline Vehicle & 846 & 18 & 4 \\
\hline Wine & 178 & 13 & 3 \\
\hline Wisconsin & 683 & 9 & 2 \\
\hline
\end{tabular}

FRM of the winning rule. Furthermore, we have selected the use of 3 labels per variable in the case of the Chi FRBCS and, for the FH-GBML algorithm, we consider the following values for the specific parameters of the genetic process:

- Number of fuzzy rules: $5 \cdot d$ rules.

- Number of rule sets: 200 rule sets.

- Crossover probability: 0.9 .

- Mutation probability: $1 / d$.

- Number of replaced rules: All rules except the best-one (Pittsburghpart, elitist approach), number of rules / 5 (GCCL-part).

- Total number of generations: 1000 generations.

- Don't care probability: 0.5.

- Probability of the application of the GCCL iteration: 0.5. 
where $d$ stands for the dimensionality of the problem (number of variables).

Finally, we indicate the values that have been considered for the parameters of the genetic tuning:

- Population Size: 50 individuals.

- Number of evaluations: 5000 - number of variables.

- Bits per gene for the Gray codification (for incest prevention): 30 bits.

The GAGRAD algorithm [19], used for comparison with our proposed methodology, considers two trapezoidal fuzzy sets per variable and represents a set of rules by a constrained neural network with the following configuration:

- The activation functions are squashing functions with $a=1 / 2$ and $\lambda=1$.

- The weights are zero or one.

- The number of hidden layers is just 1 layer.

The GAGRAD method refines the system in two steps. The first step is the rule set optimization by means of a GA and the second one is a gradient based local optimization of the fuzzy sets.

- The GA parameters are:

- Population size: 100 individuals.

- Total number of generations: 100 generations.

- Crossover probability: 0.6.

- Mutation probability: 0.02 .

- The gradient based local optimization parameters are:

$-\beta$ (power of the approximation): is increased along the process starting from 2 and stopping when is greater than 101.

- Number of hidden neurons: $4 \cdot$ number of variables.

Statistical analysis needs to be carried out in order to find significant differences among the results obtained by the studied methods [22]. We consider the use of non-parametric tests, according to the recommendations made in $[17,23]$ where it is presented a set of simple, safe and robust nonparametric tests for statistical comparisons of classifiers. In this empirical study we will apply pairwise comparisons between the algorithms using the 
Wilcoxon Signed-Ranks Test, setting at 0.05 the level of confidence $(\alpha)$ in all cases (a complete description of the test and software for its use can be found in the website: http://sci2s.ugr.es/sicidm/).

\subsection{Analysis of the IVFSs and genetic tuning performance}

The results for the Chi et al.'s method are shown in Table 2. We can observe the good behavior of IVFS model with genetic amplitude tuning with respect to the simple $\mathrm{KB}$ obtained by the original method, since it obtains the best results in most of the data-sets of the study. In order to compare both approaches, we carry out a Wilcoxon test (Table 3), which shows significant differences in favour of the model that uses IVFSs.

Table 2: Table of results for the Chi et al.'s method in Train (Tr) and Test (Tst) with their respective standard deviations

\begin{tabular}{|l|c|c|c|c|}
\hline \multirow{2}{*}{ Dataset } & \multicolumn{2}{|c|}{ Chi } & \multicolumn{2}{c|}{ Chi-IVFS-Amp } \\
\cline { 2 - 5 } & $A c_{T r}$ & $A c c_{T s t}$ & $A c c_{T r}$ & $A c c_{T s t}$ \\
\hline australian & $91.76 \pm 0.40$ & $78.55 \pm 3.50$ & $92.38 \pm 0.78$ & $\mathbf{8 3 . 3 3} \pm \mathbf{2 . 4 0}$ \\
autos & $92.55 \pm 2.15$ & $57.24 \pm 5.14$ & $94.29 \pm 2.14$ & $\mathbf{6 6 . 7 1} \pm \mathbf{7 . 6 4}$ \\
balance & $91.62 \pm 0.22$ & $89.92 \pm 1.75$ & $92.18 \pm 0.20$ & $\mathbf{9 0 . 8 8} \pm \mathbf{1 . 2 1}$ \\
bupa & $60.73 \pm 1.21$ & $\mathbf{5 7 . 6 8} \pm \mathbf{1 . 5 9}$ & $61.09 \pm 1.15$ & $\mathbf{5 7 . 6 8} \pm \mathbf{1 . 5 9}$ \\
car & $97.31 \pm 0.27$ & $76.10 \pm 3.11$ & $98.39 \pm 0.19$ & $\mathbf{9 1 . 4 4} \pm \mathbf{1 . 0 3}$ \\
cleveland & $92.22 \pm 2.15$ & $36.01 \pm 6.39$ & $92.81 \pm 2.20$ & $\mathbf{5 0 . 5 0} \pm \mathbf{6 . 4 1}$ \\
contraceptive & $52.32 \pm 1.45$ & $40.26 \pm 3.57$ & $59.44 \pm 0.66$ & $\mathbf{4 8 . 0 0} \pm \mathbf{2 . 9 6}$ \\
crx & $91.45 \pm 0.75$ & $81.78 \pm 2.56$ & $92.14 \pm 0.77$ & $\mathbf{8 5 . 4 6} \pm \mathbf{2 . 5 6}$ \\
ecoli & $76.18 \pm 1.35$ & $\mathbf{7 2 . 6 4} \pm \mathbf{7 . 0 1}$ & $77.37 \pm 0.85$ & $71.45 \pm 7.22$ \\
german & $99.47 \pm 0.10$ & $18.20 \pm 2.61$ & $99.57 \pm 0.14$ & $\mathbf{4 6 . 2 0} \pm \mathbf{2 4 . 7 1}$ \\
glass & $66.28 \pm 2.65$ & $57.95 \pm 1.34$ & $69.21 \pm 3.55$ & $\mathbf{5 9 . 8 6} \pm \mathbf{5 . 2 4}$ \\
haberman & $74.57 \pm 0.83$ & $\mathbf{7 2 . 8 8} \pm \mathbf{0 . 8 4}$ & $74.73 \pm 0.58$ & $\mathbf{7 2 . 8 8} \pm \mathbf{0 . 8 4}$ \\
hepatitis & $98.73 \pm 0.71$ & $17.50 \pm 8.15$ & $98.73 \pm 0.71$ & $\mathbf{2 0 . 0 0} \pm \mathbf{8 . 1 5}$ \\
iris & $92.94 \pm 0.75$ & $92.67 \pm 1.49$ & $97.31 \pm 0.70$ & $\mathbf{9 4 . 6 7} \pm \mathbf{2 . 9 8}$ \\
magic & $75.98 \pm 0.61$ & $74.87 \pm 1.79$ & $77.06 \pm 0.35$ & $\mathbf{7 5 . 0 8} \pm \mathbf{1 . 7 8}$ \\
newthyroid & $86.32 \pm 1.06$ & $84.65 \pm 3.89$ & $86.43 \pm 1.27$ & $\mathbf{8 5 . 1 2} \pm \mathbf{3 . 5 3}$ \\
pageblocks & $92.73 \pm 0.50$ & $\mathbf{9 1 . 4 2} \pm \mathbf{0 . 8 4}$ & $92.87 \pm 0.53$ & $\mathbf{9 1 . 4 2} \pm \mathbf{1 . 0 4}$ \\
penbased & $98.66 \pm 0.35$ & $94.27 \pm 1.59$ & $98.95 \pm 0.26$ & $\mathbf{9 4 . 3 6} \pm \mathbf{0 . 5 2}$ \\
pima & $75.45 \pm 0.72$ & $\mathbf{7 2 . 5 3} \pm \mathbf{1 . 1 3}$ & $76.30 \pm 0.75$ & $72.39 \pm 2.41$ \\
ring & $59.53 \pm 0.39$ & $\mathbf{5 2 . 7 0} \pm \mathbf{0 . 8 3}$ & $59.66 \pm 0.49$ & $52.43 \pm 1.13$ \\
tae & $61.94 \pm 3.44$ & $50.30 \pm 6.02$ & $65.44 \pm 2.24$ & $\mathbf{5 2 . 2 8} \pm \mathbf{7 . 1 7}$ \\
vehicle & $65.85 \pm 0.59$ & $\mathbf{6 0 . 8 8} \pm \mathbf{2 . 7 6}$ & $68.99 \pm 1.18$ & $60.05 \pm 3.50$ \\
wine & $98.73 \pm 0.59$ & $92.67 \pm 5.53$ & $99.86 \pm 0.31$ & $\mathbf{9 4 . 3 3} \pm \mathbf{6 . 7 1}$ \\
wisconsin & $98.17 \pm 0.29$ & $90.49 \pm 1.78$ & $98.39 \pm 0.24$ & $\mathbf{9 6 . 0 5} \pm \mathbf{1 . 6 8}$ \\
\hline Mean & $82.98 \pm 0.98$ & $67.26 \pm 3.13$ & $84.32 \pm 0.93$ & $\mathbf{7 1 . 3 6} \pm \mathbf{4 . 3 5}$ \\
\hline
\end{tabular}


Table 3: Wilcoxon's tests to compare our proposal starting from the Chi et al. method. $\mathrm{R}+$ corresponds to the sum of the ranks for the basic Chi et al.'s method and and R- to Chi with IVFS and Genetic Amplitude Tuning

\begin{tabular}{|l|c|c|c|c|}
\hline Comparison & $R^{+}$ & $R^{-}$ & Hypothesis $(\alpha=0.05)$ & p-value \\
\hline Chi vs. Chi_IVFS_amp & 35 & 265 & Rejected For Chi_IVFS_amp & 0.001 \\
\hline
\end{tabular}

Table 4: Table of results for the FH-GBML algorithm in Train (Tr) and Test (Tst) with their respective standard deviations

\begin{tabular}{|l|c|c|c|c|}
\hline \multicolumn{1}{|c|}{ Data-set } & \multicolumn{2}{|c|}{ FH-GBML } & \multicolumn{2}{c|}{ FH-GBML-IVFS-Amp } \\
\cline { 2 - 5 } & $A c c_{T r}$ & $A c c_{T s t}$ & $A c c_{T r}$ & $A c c_{T s t}$ \\
\hline australian & $87.19 \pm 0.24$ & $84.78 \pm 2.56$ & $88.42 \pm 0.93$ & $\mathbf{8 5 . 5 1} \pm \mathbf{3 . 0 3}$ \\
autos & $61.02 \pm 3.34$ & $45.34 \pm 5.48$ & $75.92 \pm 4.69$ & $\mathbf{5 4 . 7 4} \pm \mathbf{7 . 9 9}$ \\
balance & $79.04 \pm 1.36$ & $77.60 \pm 1.26$ & $80.84 \pm 1.67$ & $\mathbf{8 0 . 4 8} \pm \mathbf{2 . 3 7}$ \\
bupa & $70.62 \pm 0.83$ & $\mathbf{6 3 . 4 8} \pm \mathbf{5 . 7 4}$ & $74.33 \pm 2.27$ & $62.61 \pm 6.43$ \\
car & $75.03 \pm 2.24$ & $73.20 \pm 2.80$ & $75.33 \pm 2.24$ & $\mathbf{7 3 . 2 6} \pm \mathbf{2 . 4 0}$ \\
cleveland & $61.71 \pm 0.97$ & $54.55 \pm 2.70$ & $65.26 \pm 1.12$ & $\mathbf{5 6 . 9 1} \pm \mathbf{2 . 3 5}$ \\
contraceptive & $47.89 \pm 0.76$ & $45.22 \pm 2.51$ & $51.32 \pm 1.70$ & $\mathbf{4 8 . 2 7} \pm \mathbf{1 . 7 4}$ \\
crx & $88.30 \pm 0.48$ & $86.68 \pm 1.74$ & $88.72 \pm 0.59$ & $\mathbf{8 7 . 2 9} \pm \mathbf{2 . 4 3}$ \\
ecoli & $76.33 \pm 1.18$ & $\mathbf{7 2 . 9 1} \pm \mathbf{4 . 3 4}$ & $81.26 \pm 2.81$ & $\mathbf{7 2 . 9 1} \pm \mathbf{4 . 0 5}$ \\
german & $73.82 \pm 0.38$ & $71.30 \pm 1.82$ & $75.04 \pm 1.17$ & $\mathbf{7 1 . 6 0} \pm \mathbf{2 . 1 0}$ \\
glass & $69.10 \pm 1.77$ & $57.49 \pm 3.67$ & $74.27 \pm 2.82$ & $\mathbf{5 7 . 9 4} \pm \mathbf{2 . 3 7}$ \\
haberman & $78.26 \pm 0.98$ & $71.89 \pm 3.38$ & $78.75 \pm 1.01$ & $\mathbf{7 2 . 2 2} \pm \mathbf{2 . 3 3}$ \\
hepatitis & $91.75 \pm 1.33$ & $81.25 \pm 6.25$ & $92.06 \pm 1.59$ & $\mathbf{8 3 . 7 5} \pm \mathbf{1 0 . 4 6}$ \\
iris & $98.49 \pm 0.92$ & $95.33 \pm 4.47$ & $98.82 \pm 0.46$ & $\mathbf{9 6 . 0 0} \pm \mathbf{4 . 3 5}$ \\
magic & $79.02 \pm 1.47$ & $78.49 \pm 2.46$ & $81.19 \pm 0.78$ & $\mathbf{7 9 . 1 8} \pm \mathbf{1 . 5 6}$ \\
newthyroid & $95.32 \pm 0.41$ & $92.56 \pm 5.55$ & $97.66 \pm 1.85$ & $\mathbf{9 3 . 4 9} \pm \mathbf{3 . 0 3}$ \\
pageblocks & $95.88 \pm 0.52$ & $\mathbf{9 4 . 1 6} \pm \mathbf{1 . 2 5}$ & $96.07 \pm 0.50$ & $\mathbf{9 4 . 1 6} \pm \mathbf{1 . 2 5}$ \\
penbased & $69.83 \pm 1.99$ & $67.18 \pm 2.41$ & $83.85 \pm 1.92$ & $\mathbf{7 8 . 2 7} \pm \mathbf{2 . 7 9}$ \\
pima & $76.88 \pm 1.03$ & $73.96 \pm 1.83$ & $78.71 \pm 1.76$ & $\mathbf{7 5 . 0 0} \pm \mathbf{0 . 7 9}$ \\
ring & $84.97 \pm 1.71$ & $82.84 \pm 5.92$ & $88.43 \pm 1.61$ & $\mathbf{8 3 . 1 1} \pm \mathbf{4 . 0 0}$ \\
tae & $62.61 \pm 3.44$ & $49.01 \pm 10.90$ & $66.11 \pm 2.51$ & $\mathbf{5 2 . 3 2} \pm \mathbf{8 . 6 3}$ \\
vehicle & $61.47 \pm 1.44$ & $58.52 \pm 4.01$ & $69.46 \pm 2.10$ & $\mathbf{6 2 . 3 0} \pm \mathbf{2 . 9 9}$ \\
wine & $97.45 \pm 0.39$ & $\mathbf{9 0 . 9 7} \pm \mathbf{4 . 7 6}$ & $98.87 \pm 0.80$ & $\mathbf{9 0 . 9 7} \pm \mathbf{6 . 2 4}$ \\
wisconsin & $97.58 \pm 0.33$ & $95.61 \pm 1.63$ & $97.65 \pm 0.42$ & $\mathbf{9 5 . 7 5} \pm \mathbf{1 . 8 9}$ \\
\hline Mean & $78.31 \pm 1.23$ & $73.51 \pm 3.73$ & $81.60 \pm 1.64$ & $\mathbf{7 5 . 3 3} \pm \mathbf{3 . 6 5}$ \\
\hline
\end{tabular}

Regarding the FH-GBML approach, the results are shown in Table 4. Our approach obtains better or equal results than the basic FH-GBML algorithm in all data-sets but bupa. The statistical analysis shown in Table 5 also supports the quality of our approach, and confirms the goodness of the proposed methodology for both algorithms used in this study, since our 
Table 5: Wilcoxon's tests to compare our proposal starting from the FHGBML algorithm. $\mathrm{R}+$ corresponds to the sum of the ranks for the basic FH-GBML algorithm and and R- to FH-GBML algorithm with IVFS and Genetic Amplitude Tuning

\begin{tabular}{|l|c|c|c|c|}
\hline Comparison & $R^{+}$ & $R^{-}$ & Hypothesis $(\alpha=0.05)$ & p-value \\
\hline FH-GBML vs. FH-GBML_IVFS_amp & 17 & 183 & $\begin{array}{c}\text { Rejected For } \\
\text { FH-GBML_IVFS_amp }\end{array}$ & 0.000 \\
\hline
\end{tabular}

methodology outperforms the initial FRBCS model.

Table 6: Table of results for our proposals and GAGRAD model in Train (Tr) and Test (Tst) with their respective standard deviations

\begin{tabular}{|c|c|c|c|c|c|c|}
\hline \multirow[t]{2}{*}{ Data-set } & \multicolumn{2}{|c|}{ Chi-IVFS-Amp } & \multicolumn{2}{|c|}{ FH-GBML-IVFS-Amp } & \multicolumn{2}{|c|}{ GAGRAD } \\
\hline & $A c c_{T r}$ & $A c c_{T s t}$ & ${ }^{A C C c_{T r}}$ & $A c c_{T s t}$ & $A c c_{T}$ & $A c c_{T s t}$ \\
\hline stra & $92.38 \pm 0.78$ & $83.33 \pm 2.40$ & $88.42 \pm 0.93$ & $85.51 \pm 3.03$ & $5.62 \pm 0.55$ & $85.36 \pm 3.1$ \\
\hline & & & & & & \\
\hline & & & & & & \\
\hline & & & & 62.6 & \pm 2.69 & \\
\hline & & & 2.24 & 73.26 & $1.77 \pm 7.76$ & 60.8 \\
\hline & & & $26 \pm 1.12$ & 56.91 & $59.26 \pm 1.01$ & 10 \\
\hline & & & & 4 & & 50. \\
\hline & & & & 87. & & \\
\hline & & & & 72. & & 86 \\
\hline & & & & 71.6 & 12 & 11. \\
\hline & & & & & & \\
\hline & & & & & & \\
\hline & & & & 83. & & \\
\hline & & & & & & \\
\hline & & & & 79 & 19 & \\
\hline & 86. & & & 3 & .45 & \\
\hline & & 91.4 & 9 & 94.1 & 90.1 & 89.6 \\
\hline & & & & & 78. & 77.7 \\
\hline & & & & & & \\
\hline & & & & & & \\
\hline & & & & 52. & & 49.1 \\
\hline & & & & $62 .:$ & .46 & 597 \\
\hline & & 94.3 & 98.87 & 90.97 & $98.31 \pm 1.28$ & 95.44 \\
\hline WISCC & $98.39 \pm 0.24$ & 96.05 & $97.65 \pm 0.42$ & $95.75 \pm 1.89$ & $93.45 \pm 1.28$ & $92.97 \pm 1.76$ \\
\hline Mean & $84.32 \pm 0.93$ & $71.36 \pm 4.35$ & $81.60 \pm 1.64$ & $75.33 \pm 3.65$ & $76.36 \pm 2.46$ & $74.80 \pm 4.68$ \\
\hline
\end{tabular}

Additionally, Table 6 shows the results from SCBRDs using IVFSs with 
Genetic Amplitude Tuning for both the Chi et al.'s method and the FHGBML algorithm on one hand, and from Dombi and Gera original model [19] on the other hand. We observe that the average behavior of FH-GBML_IVFS_Amp and that of GAGRAD are good. However, our technique applied to the FHGBML algorithm obtains better results than GAGRAD in 17 out of the 24 data-sets. This superiority is clearly reflected in the statistical analysis (Table 7), where we can observe that the comparison between GAGRAD and FH-GBML with IVFS and Genetic Amplitude Tuning results in favour of this latter approach with a relatively low p-value.

Table 7: Wilcoxon's tests to compare our proposals against the GAGRAD model. $\mathrm{R}+$ corresponds to the sum of the ranks for GAGRAD method and R- to both Chi et al.'s method and FH-GBML algorithm with IVFS and Genetic Amplitude Tuning

\begin{tabular}{|l|c|c|c|c|}
\hline Comparison & $R^{+}$ & $R^{-}$ & Hypothesis $(\alpha=0.05)$ & p-value \\
\hline GAGRAD vs. Chi_IVFS_amp & 181 & 109 & Not Rejected & 0.376 \\
GAGRAD vs. FH-GBML_IVFS_amp & 99.5 & 200.5 & Not Rejected & 0.171 \\
\hline
\end{tabular}

\section{Conclusions}

In this work we have analyzed the behavior of FRBCSs, starting from a simple KB generated by any rule learning method and managing the uncertainties derived from different problems of the system by means of the IVFSs theory, which allows to have a better characterization of the fuzzy labels defining the attributes of the problem. We have adapted the FRM in order to include this representation model together with the application of a post-processing genetic tuning step in order to adjust the amplitude of the upper bound of the IVFSs.

The experimental study has determined that this methodology is a suitable solution, using as rule learning methods the Chi et al.'s and the FHGBML algorithms, and obtaining a very good performance in both cases. The achieved results showed that the proposed IVFS model with genetic amplitude tuning enhances the behavior of basic FRBCS models, outperforming the quality of the solutions obtained by standard fuzzy systems. This allows us to conclude that the introduction of IVFSs improves the behavior of algorithms which are known to perform well in the fuzzy setting. 


\section{Acknowledgment}

This work has been supported by the Spanish Ministry of Science and Technology under projects TIN2008-06681-C06-01 and TIN2007-65981.

[1] Akbarzadeh-Totonchi, M. R., Moshtagh-Khorasani, M., 2007. A hierarchical fuzzy rule-based approach to aphasia diagnosis. Journal of Biomedical Informatics 40 (5), 465-475.

[2] Alcalá, R., Alcalá-Fdez, J., Casillas, J., Cordón, O., Herrera, F., 2006. Hybrid learning models to get the interpretability-accuracy trade-off in fuzzy modeling. Soft Computing 10 (9), 717-734.

[3] Alcalá, R., Alcalá-Fdez, J., Gacto, M., Herrera, F., 2007. Rule base reduction and genetic tuning of fuzzy systems based on the linguistic 3-tuples representation. Soft Computing 11 (5), 401 - 419.

[4] Alcalá-Fdez, J., Sánchez, L., García, S., del Jesus, M., Ventura, S., Garrell, J., Otero, J., Romero, C., Bacardit, J., Rivas, V., Fernández, J., Herrera, F., 2009. KEEL: A software tool to assess evolutionary algorithms to data mining problems. Soft Computing 13 (3), 307-318.

[5] Asuncion, A., Newman, D., 2007. UCI machine learning repository. University of California, Irvine, School of Information and Computer Sciences. URL http://www.ics.uci.edu/ mlearn/MLRepository.html

[6] Bastian, A., 1994. How to handle the flexibility of linguistic variables with applications. International Journal of Uncertainty, Fuzziness and Knowledge-Based Systems 2 (4), 463-484.

[7] Bustince, H., 2000. Construction of intuitionistic fuzzy relations with predetermined properties. Fuzzy Sets and Systems 109 (3), 379-403.

[8] Bustince, H., 2000. Indicator of inclusion grade for interval-valued fuzzy sets. application to approximate reasoning based on interval-valued fuzzy sets. International Journal of Approximate Reasoning 23, 137209.

[9] Bustince, H., Herrera, F., Montero, J., 2008. Fuzzy Sets and Their Extensions: Representation, Aggregation and Models. Springer-Verlag, Berlin Heidelberg. 
[10] Bustince, H., Pagola, M., Barrenechea, E., Melo-Pinto, P., Couto, P., Tizhoosh, H. R., Montero, J., 2009. Ignorance functions. an application to the calculation of the threshold in prostate ultrasound images. Fuzzy Sets and Systems in press, doi: 10.1016/j.fss.2009.03.005.

[11] Cardoso, G., Gomide, F., 2007. Newspaper demand prediction and replacement model based on fuzzy clustering and rules. Information Sciences 177 (21), 4799 - 4809.

[12] Casillas, J., Cordón, O., Herrera, F., Magdalena, L. (Eds.), 2003. Accuracy improvements in linguistic fuzzy modeling. Springer-Verlag.

[13] Chi, Z., Yan, H., Pham, T., 1996. Fuzzy algorithms with applications to image processing and pattern recognition. World Scientific.

[14] Cordón, O., del Jesus, M. J., Herrera, F., 1999. A proposal on reasoning methods in fuzzy rule-based classification systems. International Journal of Approximate Reasoning 20 (1), 21-45.

[15] Cordón, O., Gomide, F., Herrera, F., Hoffmann, F., Magdalena, L., 2004. Ten years of genetic fuzzy systems: Current framework and new trends. Fuzzy Sets and Systems 141 (1), 5-31.

[16] Cordón, O., Herrera, F., Villar, P., 2001. A genetic learning process for the scaling factors, granularity and contexts of the fuzzy rule-based system data base. Information Sciences 136 (1-4), 85-107.

[17] Demšar, J., 2006. Statistical comparisons of classifiers over multiple data sets. Journal of Machine Learning Research 7, 1-30.

[18] Deschrijver, G., Kerre, E. E., 2007. On the position of intuitionistic fuzzy set theory in the framework of theories modelling imprecision. Information Sciences 177 (8), 1860 - 1866.

[19] Dombi, J., Gera, Z., 2008. Rule based fuzzy classification using squashing functions. Journal of Intelligent \& Fuzzy Systems 19 (1), $3-8$.

[20] Eshelman, L. J., 1991. Foundations of Genetic Algorithms. Morgan Kaufman, Ch. The CHC adaptive search algorithm: How to have safe search when engaging in nontraditional genetic recombination, pp. 265283. 
[21] Gacto, M.J., Alcalá, R., Herrera, F., 2009. Adaptation and application of multi-objective evolutionary algorithms for rule reduction and parameter tuning of fuzzy rule-based systems. Soft Computing 13 (3), 419 436

[22] García, S., Fernández, A., Luengo, J., Herrera, F., 2009. A study of statistical techniques and performance measures for genetics-based machine learning: Accuracy and interpretability. Soft Computing 13 (10), 959-977.

[23] García, S., Herrera, F., 2008. An extension on "statistical comparisons of classifiers over multiple data sets" for all pairwise comparisons. Journal of Machine Learning Research 9, 2677-2694.

[24] Gorlzakczany, M. B., 1987. A method of inference in approximate reasoning based on interval-valued fuzzy sets. Fuzzy Sets and Systems 21, $1-17$.

[25] Herrera, F., 2008. Genetic fuzzy systems: Taxonomy, current research trends and prospects. Evolutionary Intelligence 1, 27-46.

[26] Herrera, F., Lozano, M., Sánchez, A. M., 2003. A taxonomy for the crossover operator for real-coded genetic algorithms: An experimental study. International Journal of Intelligent Systems 18, 309-338.

[27] Ishibuchi, H., Nakashima, T., 2001. Effect of rule weights in fuzzy rulebased classification systems. IEEE Transactions on Fuzzy Systems 9 (4), 506-515.

[28] Ishibuchi, H., Nakashima, T., Nii, M., 2004. Classification and modeling with linguistic information granules: Advanced approaches to linguistic Data Mining. Springer-Verlag.

[29] Ishibuchi, H., Yamamoto, T., 2005. Rule weight specification in fuzzy rule-based classification systems. IEEE Transactions on Fuzzy Systems $13,428-435$.

[30] Ishibuchi, H., Yamamoto, T., Nakashima, T., 2005. Hybridization of fuzzy GBML approaches for pattern classification problems. IEEE Transactions on System, Man and Cybernetics B 35 (2), 359-365. 
[31] Klir, G., Yuan, B., 1995. Fuzzy Sets and Fuzzy Logic: Theory and Applications. Prentice Hall, Upper Saddle River, NJ.

[32] Liang, Q., Mendel, J. M., 2000. Interval type-2 fuzzy logic systems: Theory and design. IEEE Transactions on Fuzzy Systems 8, 535-549.

[33] Luengo, J., Herrera, F., 2009. Domains of competence of fuzzy rule based classification systems with data complexity measures: A case of study using a fuzzy hybrid genetic based machine learning method. Fuzzy Sets and Systems in press, doi:10.1016/j.fss.2009.04.001.

[34] Mendel, J. M., 2001. Uncertain Rule-Based Fuzzy Logic Systems. Prentice Hall, Upper Saddle River, NJ.

[35] Mendel, J. M., 2007. Advances in type-2 fuzzy sets and systems. Information Sciences 117, 84-110.

[36] Mendel, J. M., Robert, R. I. J., 2002. Type-2 fuzzy sets made simple. IEEE Transactions on Fuzzy Systems 10 (2), 117-127.

[37] Mendel, J. M., Wu, H., 2006. Type-2 fuzzistics for symmetric interval type-2 fuzzy sets: Part 1, forwarrd problems. IEEE Transactions on Fuzzy Systems 14 (6), 781-792.

[38] Nojima, Y., Ishibuchi, H., Kuwajima, I., 2009. Parallel distributed genetic fuzzy rule selection. Soft Computing 13 (3), 511 - 519

[39] Sambuc, R., 1975. Function $\phi$-flous, application a l'aide au diagnostic en pathologie thyroidienne. Ph.D. thesis, University of Marseille.

[40] Tizhoosh, H. R., 2005. Image thresholding using type-2 fuzzy sets. Pattern Recognition 38, 2363-2372.

[41] Tsang, C., Kwong, S., Wang, H., 2007. Genetic-fuzzy rule mining approach and evaluation of feature selection techniques for anomaly intrusion detection. Pattern Recognition 40 (9), 2373-2391.

[42] Turksen, I. B., 1995. Fuzzy normal forms. Fuzzy Sets and Systems 69, 319-346. 
[43] Vinterbo, S. A., Kim, E.-Y., Ohno-Machado, L., 2005. Small, fuzzy and interpretable gene expression based classifiers. Bioinformatics 21 (9), 1964-1970.

[44] Wu, D., Mendel, J. M., 2007. Uncertainty measures for interval type-2 fuzzy sets. Information Sciences 177 (23), 5378 - 5393.

[45] Zadeh, L. A., 1975. The concept of a linguistic variable and its application to approximate reasoning - i. Information Sciences 8 (3), 199 249.

[46] Zarandi, M. F., Alaeddini, A., Turksen, I., 2008. A hybrid fuzzy adaptive sampling - run rules for shewhart control charts. Information Sciences 178 (4), $1152-1170$. 